\title{
ARTICLE Cortico-thalamic hypo- and hyperconnectivity extend consistently to basal ganglia in schizophrenia
}

\author{
Mihai Avram ${ }^{1,2}$, Felix Brandl ${ }^{1,2}$, Josef Bäuml ${ }^{1,2}$ and Christian Sorg ${ }^{1,2,3}$
}

Schizophrenia is characterized by hypoconnectivity or decreased intrinsic functional connectivity (iFC) between prefrontal-limbic cortices and thalamic nuclei, as well as hyperconnectivity or increased iFC between primary-sensorimotor cortices and thalamic nuclei. However, cortico-thalamic iFC overlaps with larger, structurally defined cortico-striato-pallido-thalamo-cortical (CSPTC) circuits. If such an overlap is relevant for intrinsic hypo-/hyperconnectivity, it suggests (i) that patterns of cortico-subcortical hypo-/ hyperconnectivity extend consistently from thalamus to basal ganglia nuclei; and (ii) such consistent hypo-/hyperconnectivity might link distinctively but consonant with different symptom dimensions, namely cognitive and psychotic impairments. To test this hypothesis, 57 patients with schizophrenia and 61 healthy controls were assessed by resting-state functional magnetic resonance imaging (fMRI) and clinical-behavioral testing. IFC from intrinsic cortical networks into thalamus, striatum, and pallidum was estimated by partial correlations between fMRI time courses. In patients, the salience network covering prefrontal-limbic cortices was hypoconnected with the mediodorsal thalamus and ventral parts of striatum and pallidum; these iFC-hypoconnectivity patterns were correlated both among each other and specifically with patients' impaired cognition. In contrast, the auditorysensorimotor network covering primary-sensorimotor cortices was hyperconnected with the anterior ventral nucleus of the thalamus and dorsal parts of striatum and pallidum; these iFC-hyperconnectivity patterns were likewise correlated among each other and specifically with patients' psychotic symptoms. The results demonstrate that prefrontal-limbic hypoconnectivity and primary-sensorimotor hyperconnectivity extend consistently across subcortical nuclei and specifically across distinct symptom dimensions. Data support the model of consistent cortico-subcortical hypo-/hyperconnectivity within CSPTC circuits in schizophrenia.

Neuropsychopharmacology (2018) 43:2239-2248; https://doi.org/10.1038/s41386-018-0059-z

\section{INTRODUCTION}

Distinct intrinsic hypo- and hyperconnectivity from prefrontallimbic and primary-sensorimotor cortices with thalamic nuclei is a characteristic feature of schizophrenia [1, 2]. Intrinsic functional connectivity (iFC), i.e., coherence of ongoing infra-slow fluctuations of brain activity, which is typically measured by correlated resting-state functional magnetic resonance imaging ( $r s-f M R I)$ signal time courses, is reduced between prefrontal-limbic cortices and the mediodorsal thalamus, but increased between primarysensorimotor cortices and ventral thalamic nuclei [3-8]. This hypo-/hyperconnectivity pattern appears consistently across distinct patient groups, from ultra-high-risk to first-episode and chronic schizophrenia [9, 10], as well as unaffected siblings [11], suggesting cortico-thalamic hypo-/hyperconnectivity as an inherent feature of schizophrenia.

Cortico-thalamic intrinsic connectivity overlaps with larger cortico-striato-pallido-thalamo-cortical (CSPTC) circuits, representing structural connections between the cortex, basal ganglia, and thalamus. CSPTC circuits are organized as a series of parallelprojecting, largely segregated circuits that start in the cortex and project via the striatum, pallidum, and thalamus back to the original cortical projecting area [12]. Projections are topographically organized along circuit regions, and sub-circuits underpin preferentially distinct behavioral functions depending on the cortical origin $[13,14]$. Cortical projections of distinct functional territories (e.g., roughly limbic-emotional, associative-cognitive, primary-sensorimotor) project in an almost exclusive manner to distinct functional territories of the subcortical circuit components $[15,16]$. The overlap of distinct cortico-thalamic iFC and segregated closedloop connectivity patterns of CSPTC circuits indicates that corticothalamic iFC is shaped by, and embedded in, CSPTC circuits [17]. Critically, due to the topographical inclusion of striatum and pallidum in CSPTC circuits, such embedding of cortico-thalamic iFC suggests for schizophrenia that intrinsic hypo-/hyperconnectivity of distinct cortical regions with thalamic nuclei might be accompanied by both consonant hypo-/hyperconnectivity with basal ganglia nuclei and behavioral/cognitive impairments of the same type.

The current study tested this hypothesis, with different intrinsic brain networks as representatives of distinct cortical regions. Intrinsic brain networks (IBNs) are spatiotemporal patterns of coherent slowly fluctuating ongoing activity, which organize brain activity at large-scale level [18]. In more detail, the human cortex can be parcellated into a set of consistent IBNs [19, 20], which overlap with task-related functional networks indicating the

\footnotetext{
${ }^{1}$ Department of Neuroradiology, Klinikum rechts der Isar, Technische Universität München, Munich 81675, Germany; ${ }^{2}$ TUM-NIC Neuroimaging Center, Klinikum rechts der Isar, Technische Universität München, Munich 81675, Germany and ${ }^{3}$ Department of Psychiatry, Klinikum rechts der Isar, Technische Universität München, Munich 81675 , Germany Correspondence: Mihai Avram (mihai.avram@tum.de)

These authors contributed equally: Mihai Avram, Felix Brandl.
}

Received: 9 November 2017 Revised: 27 March 2018 Accepted: 28 March 2018

Published online: 12 April 2018 
Table 1. Participant demographics

\begin{tabular}{llll}
\hline & $\begin{array}{l}\text { Patients with } \\
\text { schizophrenia } \\
\text { (Mean; s.d.) }\end{array}$ & $\begin{array}{l}\text { Healthy } \\
\text { controls } \\
\text { (Mean; s.d.) }\end{array}$ & P \\
\hline$N$ & 57 & 61 & \\
Age [years] & $36.59(11.72)$ & $36.44(13.61)$ & 0.95 \\
Females (\%) & 26.1 & 29.89 & 0.15 \\
Age of onset [years] & $21.16(7.17)$ & - & - \\
IIIness duration [years] & $15.19(12.18)$ & - & - \\
Chlorpromazine & $349.17(317.69)$ & - & - \\
equivalents & & & $<0.05$ \\
IQ (WAIS) & $87.89(13.99)$ & $102.90(14.637)$ & $<0.05$ \\
COWAT (FAS) & $38.92(7.71)$ & $41.12(6.69)$ & - \\
PANSS (Positive) & $14.52(4.63)$ & - & - \\
\hline
\end{tabular}

Age, IQ, and verbal fluency scores were compared with two-sample $t$-tests; gender via chi-squared test

$N$ number of participants, s.d. standard deviation, WAIS Wechsler Adult Intelligence Scale, COWAT(FAS) Controlled Oral Word Association Test, letters F, A, S, PANSS Positive and Negative Syndrome Scale

functional relevance of IBNs [21, 22]. Critically, and in concordance with topographically organized cortico-thalamic and cortico-basal ganglia connections, these networks link-via iFC-distinctively with thalamic nuclei [23-25], but also with the basal ganglia $[26,27]$. Therefore, (i) distinct dysconnectivity of a given IBN with thalamic nuclei in schizophrenia might be consistently accompanied by the same type of distinct dysconnectivity with basal ganglia nuclei, and (ii) due to overlap of IBNs with task domainrelevant functional networks, functional impairments linked with cortico-thalamic dysconnectivity might extend to cortico-basal ganglia dysconnectivity.

\section{METHODS}

Overview

We used rs-fMRI and psychometric assessment data from a public database of patients with schizophrenia and healthy controls. Spatial group independent component analysis (ICA) and dual regression were performed on rs-fMRI data to identify individual, spatially independent components reflecting IBNs. Group-ICA was used to enable data-driven detection of IBNs at group level without any spatial a priori hypothesis; group-ICA was complemented by dual regression in order to detect individual IBNs; this step was used to account for known IBN changes in patients with schizophrenia [28, 29]. As IBNs of interest, we selected networks covering prefrontal-limbic and primary-sensorimotor cortices. Subsequently, partial correlations between all IBN time courses and time series of each thalamic voxel were calculated to identify specific iFC between IBNs of interest and thalamic nuclei. This step was repeated for striatum and pallidum. Resulting iFC maps were compared across groups to identify aberrant cortico-subcortical iFC in patients and whether this dysconnectivity was conserved along CSPTC circuits. Finally, we tested for correlations between significantly altered iFC in patients and cognitive impairment and positive symptoms, respectively.

Participants and clinical-behavioral scores Imaging and clinical-behavioral data of patients with schizophrenia and healthy controls were derived from an open-source database from the Center for Biomedical Research Excellence (COBRE), (http://fcon_1000.projects.nitrc.org/indi/retro/cobre.html) (for details see Table 1 and Supplementary Methods). Clinical diagnosis and behavioral assessment were based on the Structured Clinical Interview used for Diagnostic and Statistical Manual (DSM) and other psychometric tests, of which the Controlled Oral Word Association Test (COWAT, letters F, A, and S (FAS)) and Positive and Negative Syndrome Scale (PANSS) were further used in the analysis. COWAT(FAS) assesses cognitive impairment (verbal fluency and working memory) [30, 31], while PANSS quantifies the severity of positive, negative, and general symptoms in schizophrenia in distinct subscales [32].

From the original data set comprising 74 healthy participants and 72 patients with schizophrenia, we included 72 healthy controls and 71 patients in the analysis: two healthy controls (40074 and 40118) were excluded due to a history of psychological problems and one patient (40075) due to missing volumes in the rs-fMRI data (volumes 67-150).

Imaging parameters

See http://fcon_1000.projects.nitrc.org/indi/retro/cobre.html and Supplementary Methods for imaging parameters.

\section{Data preprocessing}

To characterize cortico-subcortical iFC, our data analysis followed the approach of Toulmin et al. [23], who performed single-subject space-based partial correlation analyses on cortical-subcortical iFC. For detailed description of data analysis procedures, see Supplementary Methods. In brief, preprocessing was conducted in FSL (version 5.0.9), Melodic (3.14) [33], and included realignment, coregistration to structural T1, extraction of non-brain tissue, normalization, and smoothing. Regarding head motion, we excluded 14 patients and 11 healthy controls who exhibited excessive head motion (defined as cumulative translation or rotation above $3 \mathrm{~mm}$ or $3^{\circ}$ or mean point-to-point translation or rotation above $0.15 \mathrm{~mm}$ or $0.1^{\circ}$ ). Subsequently, to further control for head motion effects across groups, we calculated the mean relative head displacement, representing the root mean squared volume-to-volume displacement for all brain voxels, calculated for the preceding volume, and tested for significant differences between the two groups [34, 35]. No differences were found regarding mean relative head motion $(p>0.5)$ in the final sample comprising 57 patients and 61 healthy controls (Table 1). To exclude residual influences of head motion at iFC level, each subject's 6 motion parameters were regressed out as covariates of no interest in subject-level partial correlation analyses (see below). Furthermore, the mean relative head motion for each subject was added as covariate of no interest in all group comparisons.

Group-ICA of rs-fMRI data, selection of intrinsic brain networks, and construction of subject-specific networks by dual regression To identify IBNs, particularly those covering prefrontal-limbic and primary-sensorimotor cortices, data were decomposed by groupICA (for details see Supplementary Methods). Automatic selection of independent components reflecting IBNs was conducted by multiple spatial cross-correlations using T-map templates provided by Yeo et al. [20], who estimated cortical organization via iFC clustering of 1000 healthy controls and found a robust parcellation of 7 IBNs. For each network, the component with the largest cross-correlation coefficient was chosen (Table S1, Figure S1). We identified components highly similar to the networks identified by Yeo et al. [20], including ventral attention, default mode (DMN), fronto-parietal (FPN), dorsal attention (DAN), limbic (LIM), visual (VIS), and auditory-sensorimotor networks (A-SM). Since the ventral attention network is often called salience network (SAL), particularly in schizophrenia literature, we followed this convention to facilitate comparisons of our results.

Following network selection, we conducted a winner-takes-all analysis of IBNs with respect to cortical voxels in order to avoid overlap between networks [23]. Subsequently, using these distinct IBN maps, we performed dual regression analysis to identify 
individual networks for each subject (for details see Supplementary Methods). Briefly, dual regression is a multivariate approach that allows the estimation of an individual version of the group-level spatial maps of ICA. Dual regression works in two stages. First, for each subject, the group-average ICA-generated set of spatial maps is regressed in a multiple regression onto the subject's 4D spacetime data set. This stage results in a set of subject-specific time courses, one per group-level spatial map. Second, those time courses are regressed in a second multiple regression, onto the same 4D data set, resulting in subject-specific spatial maps, one per group-level spatial map.

Network-specific subcortical iFC maps via partial correlation analysis and definition of network of interests

A voxel-wise partial correlation approach was employed in order to calculate subject-level iFC between the time series of each IBN and each voxel in thalamus, striatum, and pallidum, respectively (for more details see ref. [23] and Supplementary Methods). We performed these analyses in each subject's native space, assuming a more optimal detection of cortico-subcortical iFC for each subject $[23,36]$. However, independent evidence for better detection of subcortical iFC via analyses in subjects' native space is not available at the moment. For each correlation between subcortical voxels and a specific IBN, the time series of all other IBNs were regressed out, together with time series of white matter, cerebrospinal fluid, and head motion parameters [37]. The partial correlation approach yielded one correlation $(r)$ map per IBN and subcortical region for each subject, which was converted to $z$-values using Fisher's $r$-to- $z$ transformation and back-transformed into $\mathrm{MNI}$ space to enable group comparisons [38].

To specify our networks of interest, we relied on previous findings. First, based on reported prefrontal-limbic hypoconnectivity with the thalamus in schizophrenia, one network of interest should cover mainly prefrontal-limbic cortices; based on previously reported sensorimotor hyperconnectivity, the other network should cover mainly sensorimotor cortices [3-7]. Second, Tu et al. [5] demonstrated specific hypoconnectivity with the thalamus only for SAL and hyperconnectivity only for A-SM in schizophrenia. Therefore, we chose SAL and A-SM as our networks of interest for distinct hypo-/hyperconnectivity in schizophrenia.

\section{Group comparisons}

To test for within- and between-group effects, one- and twosample $t$-tests for thalamic, striatal, and pallidal $z$-maps concerning SAL and A-SM were computed at significance threshold $p<0.05$ (false discovery rate (FDR) corrected), controlled for age and gender.

Analysis of the relationship between cortico-thalamic and corticobasal ganglia iFC

In patients, Pearson's correlation analyses were performed to study the relation between averaged iFC scores of aberrant clusters in thalamus, striatum, and pallidum which presented significant hypo-/hyperconnectivity with the respective IBN. We generated masks from group difference clusters, binarized them, and extracted the averaged $z$-value from all voxels in a given cluster for each subject. This resulted in one average iFC $z$-value for each subject and cluster, with which correlations between cortico-thalamic and cortico-basal ganglia iFC were calculated. To evaluate specificity, we performed the same analysis on the same clusters also in healthy controls.

We additionally made an explorative analysis of a potential relationship between cortico-subcortical dysconnectivity and negative symptoms as measured by PANSS negative scale (for results, see Supplementary results, 2. Analysis of the relationship between cortico-subcortical iFC and negative symptoms).
Analysis of the relationship between cortico-subcortical iFC and symptom dimensions

In order to investigate the behavioral significance of dysconnectivity, we analyzed whether aberrant cortico-subcortical iFC was associated with symptom dimensions in patients. We used COWAT(FAS) as proxy for cognitive impairment and PANSS positive sub-scale for psychotic symptoms. For each cluster, the average iFC $z$-value was associated with COWAT(FAS) and PANSS positive scores by partial correlation analysis, with age, gender, IQ, and chlorpromazine equivalents (CPZ) (for the patient group only, Table 1) as covariates of no interest.

\section{RESULTS}

IBNs include prefrontal-limbic salience and auditory-sensorimotor networks

Group-ICA yielded seven independent components similar to the IBNs reported by Yeo et al. [20], namely SAL, DMN, FPN, DAN, LIM, VIS, and A-SM (Figure S1). Spatial correlation scores between components and network templates ranged from 0.33 to 0.53 (Table S1). For each component and subject, individual IBN maps were generated via dual regression. These individual IBN maps were used to define IBN-subcortical iFC for thalamus, striatum, and pallidum with SAL and A-SM, which were the basis for the following analyses regarding cortico-subcortical dysconnectivity.

Distinct hypo-/hyperconnectivity of salience and auditorysensorimotor network with the thalamus

The first goal of this study was to replicate previous findings of distinct cortico-thalamic hypo-/hyperconnectivity for IBNs covering prefrontal-limbic and primary-sensorimotor cortices, respectively $[5,9,10]$. As expected, we found hypoconnectivity between prefrontal-limbic SAL and thalamus as opposed to hyperconnectivity between primary A-SM and thalamus (Fig. 1, Table S2). In more detail, for patients, the peak of hypoconnectivity between SAL and thalamus was located in the mediodorsal nucleus $(x=14$, $y=-16, z=8$ ), while the peak of hyperconnectivity between ASM and thalamus was located in the ventral anterior nucleus $(x=$ $10, y=-6, z=4)$.

To control whether SAL hypoconnectivity was specific, we tested remaining IBNs for hypoconnectivity with the thalamus in schizophrenia. We did not find further hypoconnectivity for any IBN (Figure S4), indicating specificity of SAL hypoconnectivity with the thalamus.

Distinct patterns of hypo-/hyperconnectivity of salience and auditory-sensorimotor network with thalamus extend consistently to striatum and pallidum

To investigate whether cortico-thalamic hypo-/hyperconnectivity of SAL and A-SM extends consistently to the basal ganglia, we repeated the partial correlation iFC analysis for striatum and pallidum (Fig. 1, Table S2). Remarkably, the directions of corticobasal ganglia dysconnectivity were consistent with corticothalamic dysconnectivity. Both SAL-striatum and SAL-pallidum $\mathrm{iFC}$ were reduced in patients, peaking in right ventral putamen $(x$ $=20, y=16, z=-10)$ and right ventral pallidum $(x=20, y=-12$, $z=-6)$, respectively, while A-SM-striatum and A-SM-pallidum iFC were increased, with peaks in right dorsal caudate $(x=16, y=$ $-14, z=22)$ and right dorsal pallidum $(x=18, y=-6, z=4)$, respectively. This finding suggests that schizophrenia is characterized by hypoconnectivity between SAL and mediodorsal thalamus, ventral putamen, and ventral pallidum, as opposed to hyperconnectivity between A-SM and ventral anterior thalamus, dorsal caudate, and dorsal pallidum.

Next, we tested whether cortico-subcortical dysconnectivity is confounded by antipsychotic medication. Therefore, we computed Pearson's correlations between $\mathrm{CPZ}$ and subcortical connectivity of SAL and A-SM, respectively, for each subcortical 


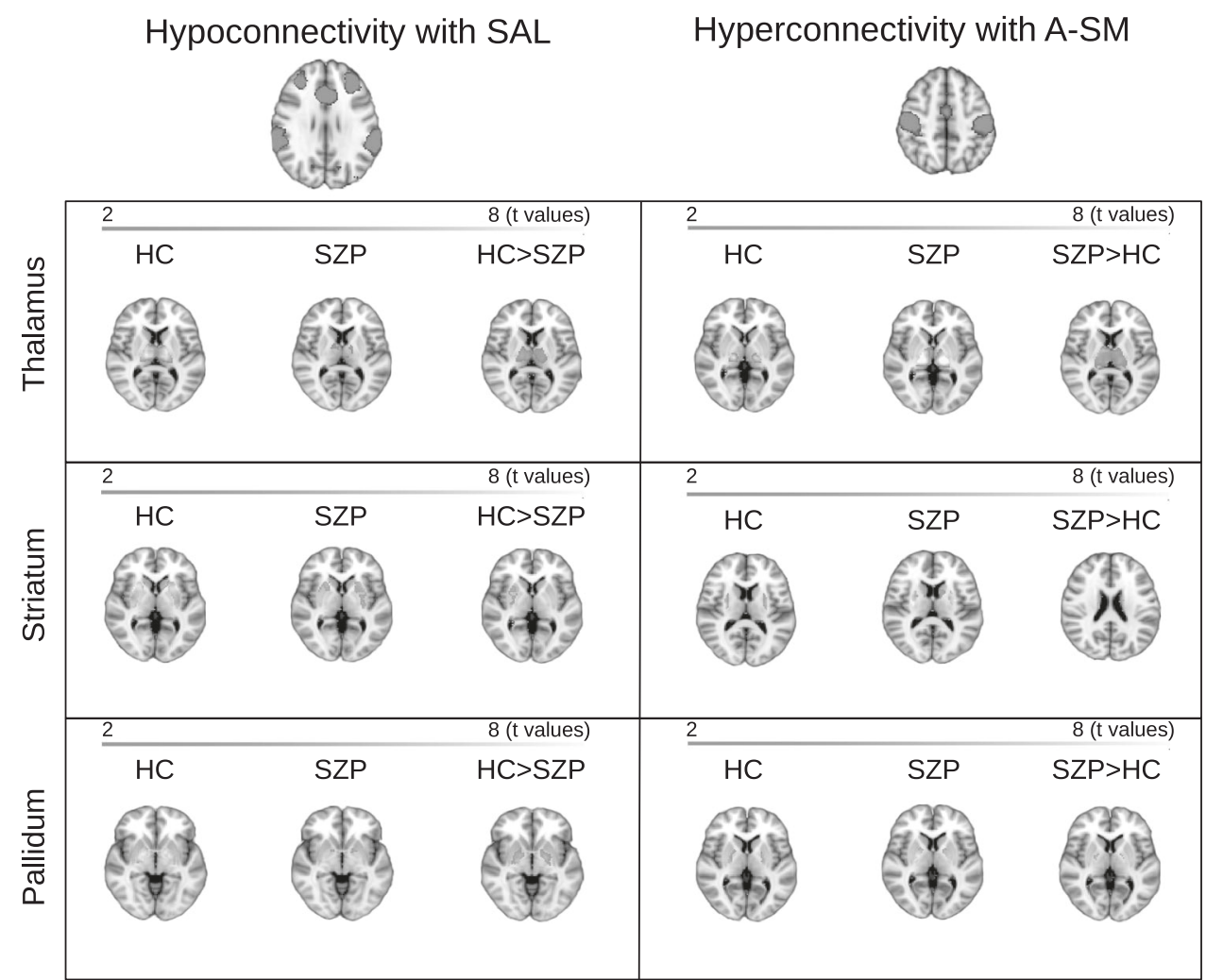

Fig. 1 Cortico-subcortical dysconnectivity for SAL and A-SM in schizophrenia. Top Hypo-/hyperconnectivity between SAL and A-SM and thalamus. Middle Hypo-/hyperconnectivity between SAL and A-SM and striatum. Bottom Hypo-/hyperconnectivity between SAL and A-SM and pallidum. Left (all panels): iFC between corresponding IBN and subcortical structure for patients and controls, respectively (voxel-wise one-sample $t$-tests, $p<0.05$, FWE-corrected). Right (all panels): between-group differences for iFC between corresponding IBN and subcortical structure (voxel-wise two-sample $t$-tests, $p<0.05$, FDR-corrected). The color bar indicates $t$-values, with brighter colors representing higher $t$ values. HC healthy controls, SZP patients with schizophrenia, SAL salience network, A-SM auditory/sensorimotor network

region in patients. No significant correlation was found for any cortico-subcortical connectivity $(p>0.05)$, suggesting that corticosubcortical dysconnectivity of patients is independent from medication.

Subsequently, we tested whether cortico-thalamic and corticobasal ganglia dysconnectivity were related in patients (Fig. 2). Cortico-thalamic and cortico-basal ganglia hypoconnectivity of SAL were highly and significantly correlated across patients (cortico-thalamic with cortico-striatal iFC: $r=0.66, p<0.001$; cortico-thalamic with cortico-pallidal iFC: $r=0.61, p<0.001)$. The same was found for A-SM hyperconnectivity (cortico-thalamic with cortico-striatal iFC: $r=0.58, p<0.001$; cortico-thalamic with cortico-pallidal iFC: $r=0.30, p=0.01)$. Moreover, similar results were found for healthy controls for SAL (cortico-thalamic with cortico-striatal iFC: $r=0.60, p<0.001$; cortico-thalamic with cortico-pallidal iFC: $r=0.61, p<0.001)$ and A-SM connectivity (cortico-thalamic with cortico-striatal iFC: $r=0.65, p<0.001$; cortico-thalamic with cortico-pallidal iFC: $r=0.68, p<0.001$ ) (Figure S2). This consistency of correlated cortico-thalamic and cortico-basal ganglia iFC across networks and groups suggests that distinct cortico-thalamic and cortico-basal ganglia connectivity patterns are part of distinct CSPTC circuits.

To control whether aberrant IBN-subcortical region iFC was confounded by aberrant global IBN connectivity, we performed a control analysis accounting for IBN global connectivity. We calculated an index of global connectivity for each IBN and participant, based on IBN inter-network connectivity. In more detail, for each of the 7 IBNs we correlated the time courses from one IBN to all other IBNs, generating 21 correlation coefficients, per participant, which were then averaged, resulting in one connectivity index per IBN and subject. Subsequently, we computed partial correlations in which we correlated SALthalamic with SAL-striatal and SAL-pallidal iFC, while controlling for the IBN global connectivity index. We repeated the procedure for A-SM. We found that for each IBN, cortico-subcortical iFC remains highly correlated across distinct subcortical structures, i.e., cortico-thalamic and cortico-basal ganglia hypoconnectivity of SAL were highly and significantly correlated across patients (cortico-thalamic with cortico-striatal iFC: $r=0.65, p<0.001$; cortico-thalamic with cortico-pallidal iFC: $r=0.65, p<0.001)$. The same was found for A-SM hyperconnectivity (cortico-thalamic with cortico-striatal iFC: $r=0.65, p<0.001$; cortico-thalamic with cortico-pallidal iFC: $r=0.59, p<0.001)$. Therefore, IBN global connectivity does not seem to account for the correlations between cortico-thalamic with cortico-basal ganglia iFC for neither SAL nor A-SM.

Consistent association between hypo-/hyperconnectivity and symptom dimensions across subcortical nuclei

Behavioral relevance of cortico-subcortical dysconnectivity was investigated with a multivariate model incorporating all factors. In more detail, we computed a multivariate analysis of variance, with COWAS and PANSS+ as dependent variables, and included all other factors (i.e., cortico-subcortical iFC and nuisance variables) as covariates in the model. We found the multivariate model to be significant for both COWAT $(F(10,42)=4.80, p<0.001)$ and PANSS $+(F(10,42)=2.35, p=0.02)$. Further, we found a significant association between A-SM-pallidum iFC and PANSS + $(F(1,42)=$ $12.15, p=0.001)$ as well as for SAL-thalamic iFC and COWAT(FAS) $(F(1,42)=3.55, p=0.04)$. Since for each IBN, iFC with distinct subcortical regions are highly correlated among each other (Fig. 2), we tested whether shared variance in IBN-subcortical iFC across 

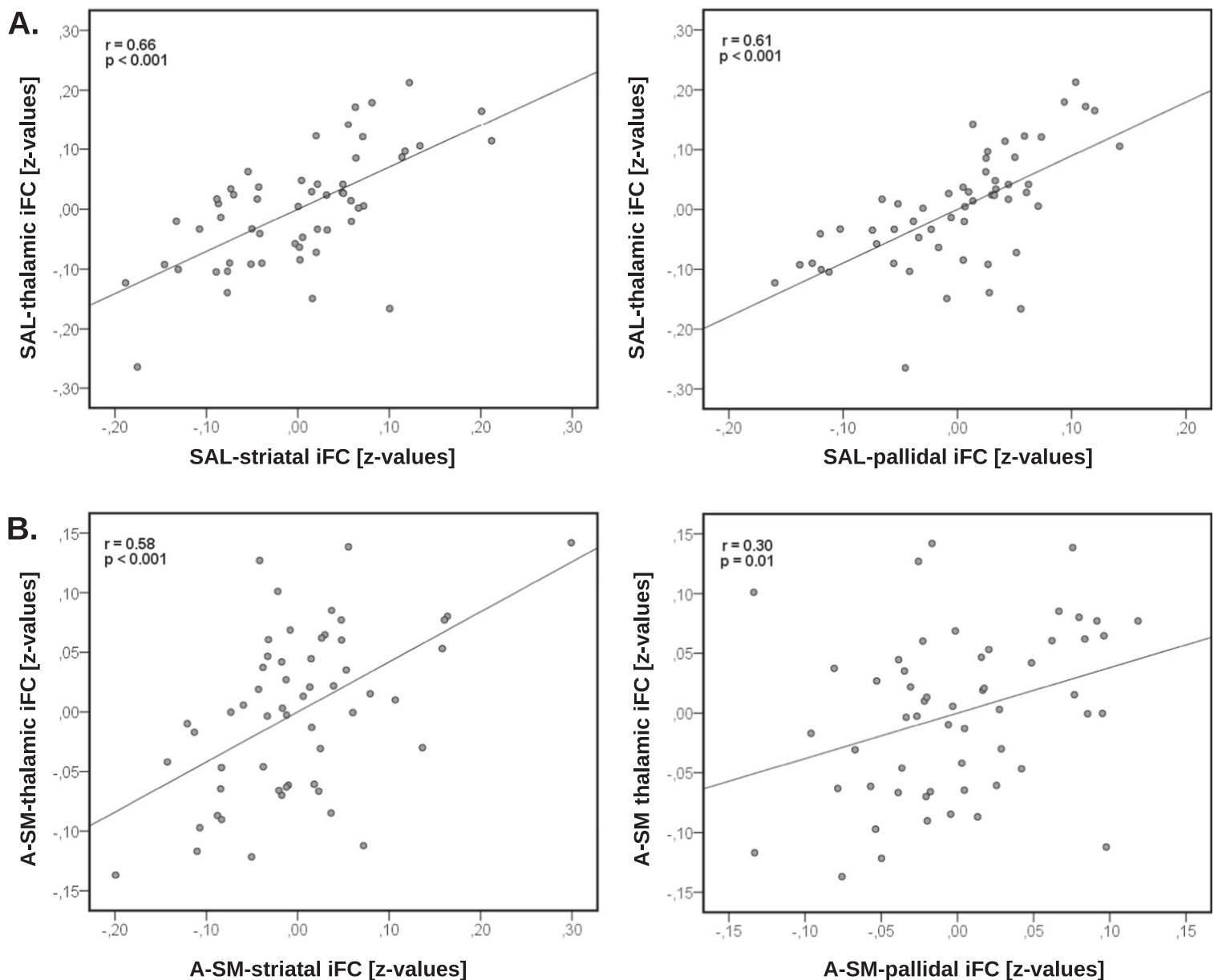

Fig. 2 Correlations of cortico-thalamic and cortico-basal ganglia iFC in patients. A Pearson's correlations of SAL-thalamic iFC with SAL-striatal (left) and SAL-pallidal iFC (right). B A-SM-thalamic correlations with A-SM-striatal (left) and A-SM-pallidal iFC (right). iFC intrinsic functional connectivity, SAL salience network, A-SM auditory/sensorimotor network, $r$ Pearson's correlation coefficient

striatum, pallidum, and thalamus explains various symptom dimensions distinctively for SAL and A-SM. We performed a principal component analysis on cortico-subcortical hypoconnectivity (SAL-thalamic, SAL-striatal, and SAL-pallidal iFC) and put the extracted Eigenvalues of 'shared' dysconnectivity into partial correlation analyses for COWAS and PNASS + with age, gender, IQ, and medication as nuisance variables. We found a significant positive association between hypoconnectivity Eigenvalues and COWAT(FAS) $(r=0.43, p=0.002)$ as well as a positive association between A-SM Eigenvalues and PANSS+ scores $(r=0.47, p<$ 0.001 ). This result indicates that SAL-subcortical hypoconnectivity and A-SM-subcortical hyperconnectivity links distinctively with cognitive impairment and psychotic symptoms. To present the link between single IBN-subcortical region iFC and symptom dimensions for given IBN and subcortical region, we repeated the just described partial correlation analysis for this IBN-subcortical region iFC (Fig. 3, Table S3). First, we tested for associations between cortico-subcortical hypoconnectivity in patients and cognitive impairments and psychotic symptoms, respectively. We found a significant positive correlation between COWAT(FAS) scores and SAL-thalamic hypoconnectivity $(r=0.36, p=0.006)$ and, correspondingly, both SAL-striatal $(r=0.36, p=0.006)$ and SAL-pallidal ( $r=0.26, p=0.03$ ) hypoconnectivity (Fig. 2). No significant correlation was found for SAL-subcortical hypoconnectivity and psychotic symptoms for thalamus $(r=0.03, p=0.81)$, striatum $(r=-0.09, p=0.51)$, or pallidum $(r=-0.05, p=0.71)$, suggesting a specific link between SAL-subcortical hypoconnectivity and cognitive impairment. Furthermore, no significant associations between SAL-subcortical iFC and COWAT(FAS) scores were found for healthy controls-analyzed separately, suggesting specificity for patients and dysconnectivity (Figure S3). Additionally, we tested whether the association between SAL-subcortical hypoconnectivity and cognitive scores extended to other cognitive measures beyond COWAT(FAS). We found such associations for two additional cognitive measures, namely Matrics working memory scale and Wechsler memory scale III, indicating that SAL-subcortical hypoconnectivity is relevant for impaired cognition independent of the applied cognitive measure (for further details see Supplementary results).

Second, we tested for associations between cortico-subcortical hyperconnectivity in patients and cognitive impairment and psychotic symptoms, respectively. Significant positive correlations were found between PANSS positive scale and A-SM-thalamus hyperconnectivity $(r=0.26, p=0.03)$ and, correspondingly, both A-SM-striatum $(r=0.25, p=0.04)$ and A-SM-pallidum $(r=0.54$, $p<0.001$ ) hyperconnectivity (Table S3, Fig. 3). No associations were found between A-SM-subcortical hyperconnectivity and cognitive impairment for striatum $(r=-0.22, p=0.11)$ or pallidum $(r=-0.20, p=0.15)$, and only a trend for thalamus $(r=-0.25$, $p=0.06)$. No significant associations between A-SM-subcortical iFC and COWAT(FAS) scores were found for healthy controls (Figure S3). These results suggest a specific link between A-SM-subcortical hyperconnectivity and psychotic symptoms in patients. 
A.

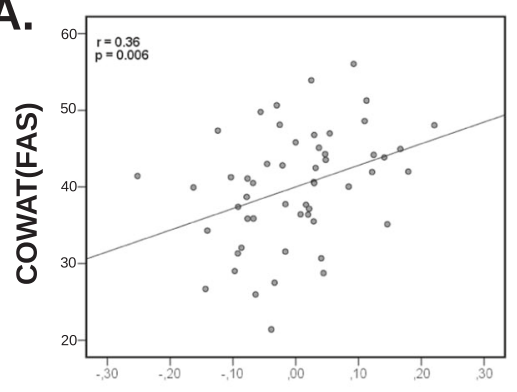

SAL-thalamic iFC [z-values]

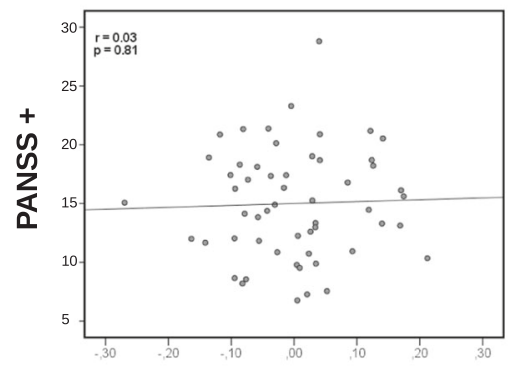

SAL-thalamic iFC [z-values]

B.
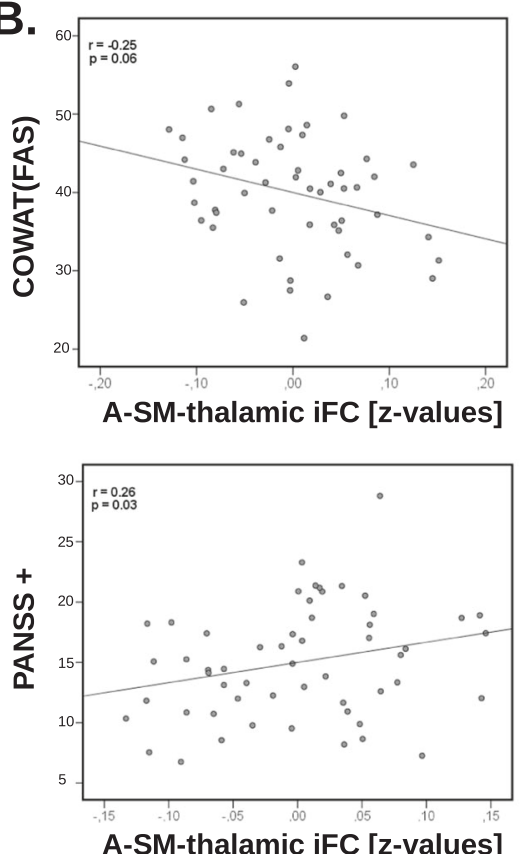

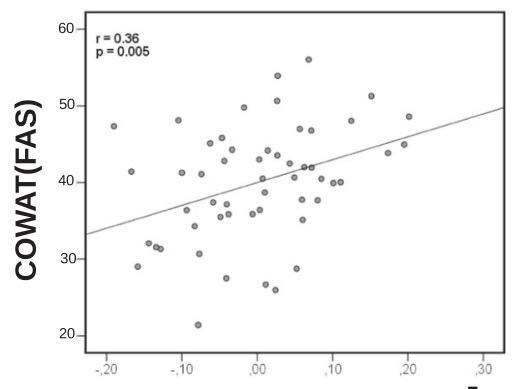

SAL-striatal iFC [z-values]
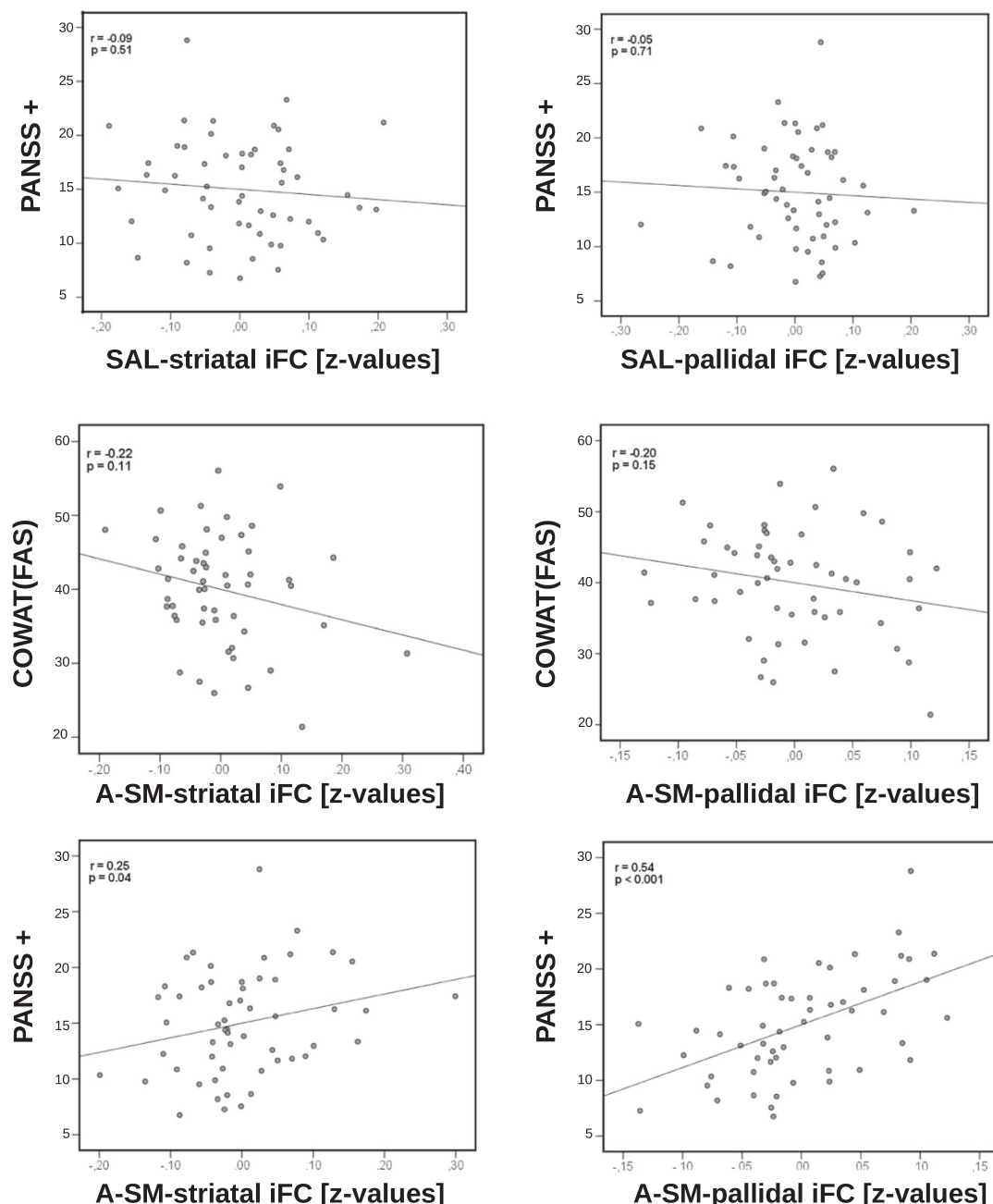

A-SM-pallidal iFC [z-values]

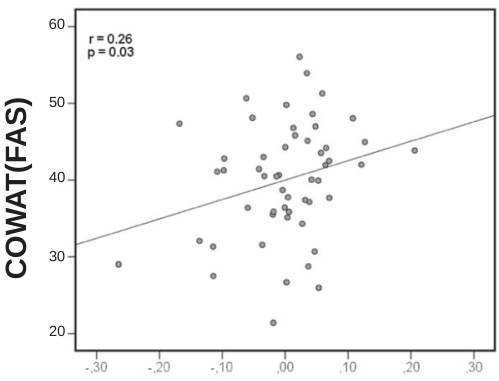

SAL-pallidal iFC [z-values]

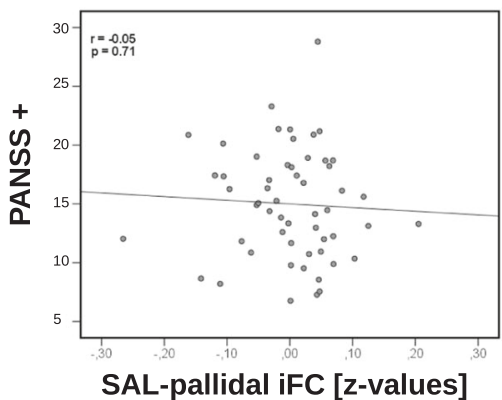

SAL-pallidal iFC [z-values]

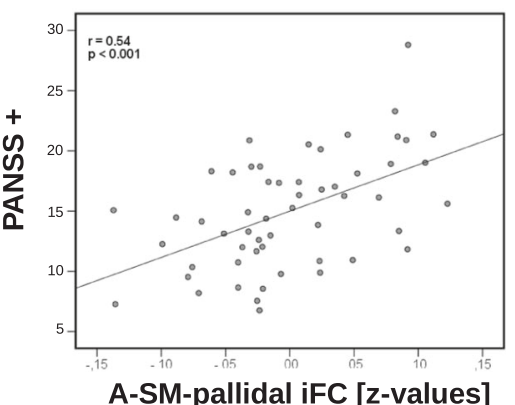

Fig. 3 Cortico-subcortical dysconnectivity is distinctively associated with symptom dimensions in schizophrenia. A Pearson's correlations between iFC of SAL-hypoconnectivity clusters (thalamus, striatum, pallidum) and COWAT(FAS) (top) and PANSS positive scale (bottom). B Pearson's correlations between iFC of A-SM-hyperconnectivity clusters (thalamus, striatum, pallidum) and COWAT(FAS) (top) and PANSS positive scale (bottom). iFC intrinsic functional connectivity, SAL salience network, A-SM auditory/sensorimotor network

\section{DISCUSSION}

Using rs-fMRI and clinical-behavioral assessments, we tested whether in schizophrenia, prefrontal-limbic hypoconnectivity and primary-sensorimotor hyperconnectivity with thalamic nuclei extend consistently to basal ganglia nuclei and symptom dimensions. First, we found consistent and inter-correlated hypoconnectivity between prefrontal-limbic salience network and thalamus, striatum, and pallidum, respectively, as well as consistent and inter-correlated hyperconnectivity between auditory-sensorimotor network and the same subcortical structures. Second, SAL cortico- subcortical hypoconnectivity was specifically linked with cognitive impairment but not psychotic symptoms, while A-SM corticosubcortical hyperconnectivity was associated with psychotic symptoms but not cognitive impairment. These results relate-to our knowledge for the first time-previous findings of distinct cortico-thalamic dysconnectivity in schizophrenia in a consistent way with distinct cortico-basal ganglia dysconnectivity and specific behavioral impairments. In summary, data support a model of consistent cortico-subcortical hypo- and hyperconnectivity within CSPTC circuits in schizophrenia. 
Cortico-thalamic hypo-/hyperconnectivity extends consistently to basal ganglia and symptom dimensions

We found that cortico-thalamic and cortico-basal ganglia hypo-/ hyperconnectivity of the same intrinsic brain network were consistent across subcortical nuclei in schizophrenia (Figs. 1 and 2). Prefrontal-limbic hypoconnectivity of SAL with the dorsomedial thalamus extends consistently to cortico-striatal and corticopallido hypoconnectivity in the ventral putamen and ventral pallidum, respectively, in patients (Fig. 1), while primarysensorimotor hyperconnectivity of A-SM with the ventral thalamus extends consistently to cortico-striatal and cortico-pallido hypoconnectivity in the dorsal caudate and dorsal pallidum (Fig. 1). Furthermore, both distinct cortical hypoconnectivities and hyperconnectivities were correlated among each other for different subcortical nuclei across patients, supporting the notion of consistent cortico-subcortical hypo-/hyperconnectivity in schizophrenia (Fig. 2). Single dysconnectivities such as prefrontal-limbic hypoconnectivity with the dorsomedial thalamus replicate (for thalamus and striatum) or extend (for the pallidum) previous findings and are discussed in detail in the supplement (Supplement Discussion).

Furthermore, we found that distinct cortico-subcortical hypo-/ hyperconnectivity was consistently linked with different symptom dimensions (Fig. 3, Table S3). SAL hypoconnectivity with thalamus, striatum, and pallidum was specifically associated with cognitive impairment in terms of reduced verbal fluency, but not psychotic symptoms measured by the PANSS+ scale, while A-SM hyperconnectivity with subcortical nuclei was specifically associated with psychotic symptoms, but not with cognitive impairment. SAL has a central role in the detection of behaviorally relevant stimuli and flexible control of goal-directed behavior [39], being relevant for cognitive control and working memory in general, and verbal fluency in particular [40-42]. Consistent with our findings, several studies have linked aberrant SAL-iFC with thalamus and basal ganglia to verbal fluency and working memory impairments in schizophrenia [43-45]. Similarly, Giraldo-Chica et al. [46] found a specific association between reduced prefrontal-thalamic structural connectivity and working memory in patients with schizophrenia. In contrast, previous findings about the link between sensorimotor cortices and associated subcortical connectivity with psychotic symptoms are mixed (for review see Giraldo-Chica and Woodward [1]). For instance, Anticevic et al. [3] reported an association between sensorimotor cortico-thalamic hyperconnectivity and general psychopathology, Cheng et al. [47] with negative symptoms, and other groups found no association with clinical assessments $[5,8,10]$. Concerning striatum, hyperconnectivity with auditory cortices has been linked to auditory hallucinations, a key positive symptom in schizophrenia [48]. Intrinsic brain networks such as SAL and A-SM parcellate the cortex into ensembles of coherent ongoing brain activity [20,49], with this parcellation extending to basal ganglia and thalamus via cortico-subcortical iFC [23, 26]. Furthermore, the IBN-based parcellation overlaps with task domain-based parcellations of functional networks [21, 50]; for example, as mentioned above, SAL is consistently involved in cognitive control. Our finding of consistent IBN-subcortical dysconnectivity and symptom dimension corresponds with this task domain-relevant parcellation of functional network based cortico-subcortical connectivity.

Cortico-subcortical hypo-/hyperconnectivity is embedded within CSPTC circuits

Consistent cortico-subcortical hypo-/hyperconnectivity suggests that consonant cortico-striatal/pallidal/thalamic dysconnectivity of schizophrenia reflects consistent dysconnectivity within larger closed feedback CSPTC circuits, which are defined by structural connectivity $[13,14]$. (i) We found that network-specific subcortical $\mathrm{iFC}$ of distinct subcortical nuclei is correlated among them not only in patients (Fig. 2) but also in healthy controls (Figure S2). This indicates the strong link between cortico-striatal, cortico-pallidal, and cortico-thalamic iFC for a given cortical region or network, and supports the notion that such cortico-subcortical iFC is embedded in CSPTC circuits. (ii) We found that peaks of iFC changes in patients between SAL and basal ganglia and thalamus matched a specific CSPTC circuit, namely the limbic/emotional circuit (for more details see below), with peaks in ventral putamen, ventral pallidum, and dorsomedial thalamus. Likewise, peaks of iFC changes between A-SM and basal ganglia and thalamus matched another specific CSPTC circuit, namely the sensorimotor circuit (see below), with peaks in dorsal caudate, dorsal pallidum, and ventral thalamus (Fig. 1). This correspondence between cortico-subcortical iFC changes and distinct CSPTC circuits suggests that consistent cortico-subcortical dysconnectivity reflects distinct impairments in different CSPTC circuits. (iii) CSPTC circuits have characteristic topographic and functional organization, which corresponds with distinct IBN-based cortico-subcortical dysconnectivity in schizophrenia. In more detail, the output of a given cortical sub-region of a CSPTC circuit, mainly from layer 5 pyramidal cells, to striatum, pallidum, and thalamus is topographically organized. Topographic CSPTC circuits' projections segregate into functional circuits, largely defined by the functionality of the originating cortical region [51]. For instance, a typical limbic/emotional circuit originates in the anterior cingulate cortex (ACC) and projects to ventral striatum, followed by ventral pallidum, mediodorsal nucleus in the thalamus, and finally back to ACC, while central cortices of sensorimotor function project more caudally to dorsal striatum and mainly putamen, followed by dorsal pallidum, 'sensorimotor' nuclei of the thalamus such as anterior ventral nuclei, and finally back to cortical 'sensorimotor' areas [14, 52]. Our findings of IBN-based normal and aberrant cortico-striatal/pallidal/thalamic iFC in controls and patients corresponds with this topographic and functional organization of CSPTC circuits, suggesting that iFC-based (dys)connectivity patterns reflect CSPTC circuits. In line with these results, a recent study assessed cortico-thalamic connectivity by parcellating the human thalamus and linking these parcels with functional and structural connectivity to the cortex [53]. Despite the different approach, Kumar et al. [53] also found iFC between prefrontal association areas corresponding to parts of SAL and thalamic mediodorsal nuclei, as well as somatosensory areas and the ventral anterior nuclei of the thalamus.

Possible neural mechanisms underlying consistent corticosubcortical dysconnectivity in schizophrenia

Dysconnectivity of specific cortical networks across several subcortical nuclei might reflect distinct pathophysiological changes along distinct parts of CSPTC circuits, namely cortical and subcortical regions. (i) Concerning cortical pathophysiology, recent studies show and propose several cortical cellular mechanisms that might be changed in schizophrenia, resulting in imbalanced excitatory and inhibitory activity of infragranular output [54-56]. For example, multiple pre- and postsynaptic abnormalities in GABAergic parvalbumin-containing interneurons, which weaken inhibitory control of layer $2 / 3$ neurons on cortical output layers 5, have been associated with schizophrenia [56-58]. Based on assumed cortical NMDA-receptor hypofunction, a recent study demonstrated a specific link between cortical microcircuitry pathophysiology and large-scale iFC systems [59]. More specifically, in a microcircuit model-based approach, Yang et al. [59] showed that selective iFC dysconnectivity within associative and primary-sensorimotor networks was linked to cortical imbalances of excitatory and inhibitory activity. However, interpreting our results in terms of cortical pathophysiology is complicated by several factors, including the complexity of CSPTC circuitry and resting-state fMRI signal. First, CSPTC circuitry is difficult to disentangle due to both projection type (i.e., unidirectionalsuch as cortico-striatal-and bidirectional projections-cortico- 
thalamic/thalamo-cortical), and additional direct cortico-thalamic connections, which has been shown to influence CSPTC circuitry [60]. Second, it is difficult to link cellular cortical pathology to resting-state $\mathrm{fMRl}$, which is limited by both spatial resolution and lack of directionality.

(ii) Subcortical nuclei disturbances might also contribute or generate impaired cortico-subcortical iFC. Concerning thalamus as a potential source of dysconnectivity for instance, recent evidence suggests that thalamic neurons exhibit infra-slow fluctuations between 0.01 and $0.1 \mathrm{~Hz}$, which are in the same range as intrinsic fluctuations measured by rs-fMRl, and may as such contribute to cortico-thalamic iFC [61]. Based on such evidence, the thalamus, and in particular the mediodorsal nucleus, is thought to gate cortico-cortical iFC [62-64]. Therefore, disturbances of these functions in schizophrenia, particularly in the mediodorsal nucleus, could contribute to altered cortico-thalamic iFC. This idea is also supported by post-mortem studies showing that thalamic inputs to the cortex are reduced in schizophrenia, suggesting that at least some iFC aberrations could be related to thalamic pathology [65]. Concerning the striatum as a potential source of cortico-subcortical dysconnectivity, altered striatal dopamine levels could play an important role: striatal dopamine levels are consistently elevated in patients with schizophrenia [66] and enhanced dopamine has been suggested to increase frontostriatal iFC [67]. Therefore, striatal dopamine dysfunction could contribute to cortico-striatal dysconnectivity in schizophrenia.

\section{STRENGTHS AND LIMITATIONS}

The current study has some strengths and limitations. First, to analyze cortico-subcortical iFC, we used IBNs as cortical sources of iFC. Next, to analyze cortico-subcortical iFC, we used ICA-based IBNs as cortical seeds of iFC. Several points have to be made explicit regarding this ICA-based-IBN approach. (i) In contrast to regions or lobes as cortical seeds of iFC, IBN seeds reflect a 'natural' parcellation of the cortex based on the coherence of ongoing cortical slowly fluctuating activity, with such parcellation being on the one hand highly robust across individuals (including patients) and states [20], on the other hand being detectable in a data-driven way without previous a priori assumptions [20]. Furthermore, IBNs represent a reasonable trade-off for the size of cortical sources: using anatomically (thus hypothesis-free) defined whole lobes as iFC sources would bear the risk that cortical representative time courses are mixed and unspecific, while using smaller regions as sources might be more specific but requires a priori assumptions for region definition [29]. However, group-ICA-based IBN detection might also induce some confounds on aberrant cortico-subcortical connectivity. IBN parcellation assumes relatively homogenous blood oxygen level dependent (BOLD) fluctuations within IBNs across patients and controls; however, patients with schizophrenia show aberrant segregation and integration within and across IBNs [29], suggesting aberrant homogeneity of BOLD fluctuations within cortical seed IBNs. Such BOLD non-homogeneity might confound aberrant IBN subcortical connectivity. To account for such individual variability of IBNs, we complemented group-ICA-based parcellation with a dual regression approach, which provides more 'individual' versions of IBN time courses and spatial outlines. Nevertheless, one should be aware that observed IBN dysconnectivity to subcortical regions might include aberrant IBN homogeneity. (ii) IBN parcellation is reflected by corresponding organization in subcortical nuclei such as striatum [26] and thalamus [23] via cortico-subcortical iFC; however, one has to be aware that this assumption of IBN-compatible subcortical organization has not been shown for the pallidum yet, nor for the thalamus, based on the IBNs defined by Yeo et al. [20]. Therefore, one should have in mind that definitive evidence for IBN-based pallidum organization is missing. Second, we defined our main outcome measures (i.e., IBNs for the seed-based analysis) in each subject's native space following the approach of refs. [23, 37]. Although such approaches might lead to a more reliable definition of regions of interest (ROls, particularly subcortical ROls) in each individual's anatomy than the canonical approach (i.e., in $\mathrm{MNI}$ space), likely resulting in improved detection of individual iFC, this has not been shown explicitly for resting-state fMRI. At the moment, only indirect evidence for the superiority of our approach is available, based on task-fMRI [36]. Third, regarding data sample, the patient group included patients with varying severity of psychotic symptoms and number of psychotic episodes, and therefore it is unclear whether the observed dysconnectivity is related to the disorder's status (chronic, acute, remission) or represents a permanent feature of schizophrenia [9, 11]. Fourth, despite controlling for medication via chlorpromazine equivalents, we cannot exclude medication effect confounders. It is well known that antipsychotics influence iFC, and therefore our results should be interpreted carefully $[68,69]$. Future studies may address this issue by investigating corticosubcortical iFC in unmedicated patients. Finally, the duration of the rs-fMRI acquisition is relatively short. Although several rs-fMRI studies have shown that a scan time in the range of 5-7 min results in the acquisition of stable IBNs [70-72], a longer duration beyond $13 \mathrm{~min}$ can increase the reliability of iFC [73].

\section{CONCLUSION}

Both prefrontal-limbic hypoconnectivity and primarysensorimotor hyperconnectivity in schizophrenia extend consistently across thalamus, striatum, and pallidum, and link specifically with impaired cognition and psychotic symptoms, respectively. These results suggest that cortico-thalamic and cortico-basal ganglia dysconnectivity are integrated in a consistent way-i.e., for direction of change, subcortical nuclei, and associated symptom dimension-in larger cortico-striato-pallido-thalamocortical circuits.

\section{ACKNOWLEDGEMENTS}

We thank the 1000 Functional Connectomes project for contributing data and organizing its dissemination. This work has been supported by the European Union 7th Framework Programme, TRIMAGE-a dedicated trimodality (PET/MR/EEG) imaging tool for schizophrenia (grant no. 602621).

\section{ADDITIONAL INFORMATION}

Supplementary Information accompanies this paper at https://doi.org/10.1038/ s41386-018-0059-z

Competing interests: The authors declare no competing interests.

Publisher's note: Springer Nature remains neutral with regard to jurisdictional claims in published maps and institutional affiliations.

\section{REFERENCES}

1. Giraldo-Chica M, Woodward ND. Review of thalamocortical resting-state fMRI studies in schizophrenia. Schizophr Res. 2017;180:58-63.

2. Murray JD, Anticevic A. Toward understanding thalamocortical dysfunction in schizophrenia through computational models of neural circuit dynamics. Schizophr Res. 2017;180:70-77.

3. Anticevic A, Cole MW, Repovs G, Murray JD, Brumbaugh MS, Winkler AM, et al. Characterizing thalamo-cortical disturbances in schizophrenia and bipolar illness. Cereb Cortex. 2014;24:3116-30.

4. Anticevic A, Yang G, Savic A, Murray JD, Cole MW, Repovs G, et al. Mediodorsal and visual thalamic connectivity differ in schizophrenia and bipolar disorder with and without psychosis history. Schizophr Bull. 2014;40:1227-43.

5. Tu PC, Lee YC, Chen YS, Hsu JW, Li CT, Su TP. Network-specific cortico-thalamic dysconnection in schizophrenia revealed by intrinsic functional connectivity analyses. Schizophr Res. 2015;166:137-43. 
6. Welsh RC, Chen AC, Taylor SF. Low-frequency BOLD fluctuations demonstrate altered thalamocortical connectivity in schizophrenia. Schizophr Bull. 2010;36:713-22.

7. Woodward ND, Heckers S. Mapping thalamocortical functional connectivity in chronic and early stages of psychotic disorders. Biol Psychiatry. 2016;79:1016-25.

8. Woodward ND, Karbasforoushan $\mathrm{H}$, Heckers S. Thalamocortical dysconnectivity in schizophrenia. Am J Psychiatry. 2012;169:1092-9.

9. Anticevic A, Haut K, Murray JD, Repovs G, Yang GJ, Diehl C, et al. Association of thalamic dysconnectivity and conversion to psychosis in youth and young adults at elevated clinical risk. JAMA Psychiatry. 2015;72:882-91.

10. Woodward ND, Heckers S. Mapping thalamocortical functional connectivity in chronic and early stages of psychotic disorders. Biol Psychiatry. 2016;79:1016-25.

11. Lui S, Yao L, Xiao Y, Keedy SK, Reilly JL, Keefe RS, et al. Resting-state brain function in schizophrenia and psychotic bipolar probands and their first-degree relatives. Psychol Med. 2015;45:97-108.

12. Alexander GE, DeLong MR, Strick PL. Parallel organization of functionally segregated circuits linking basal ganglia and cortex. Annu Rev Neurosci. 1986;9:357-81.

13. Haber SN. The primate basal ganglia: parallel and integrative networks. J Chem Neuroanat. 2003;26:317-30.

14. Haber SN. Corticostriatal circuitry. Dialog Clin Neurosci. 2016;18:7-21.

15. Eid L, Parent M. Chemical anatomy of pallidal afferents in primates. Brain Struct Funct. 2016;221:4291-317.

16. Parent A, Hazrati LN. Functional anatomy of the basal ganglia. I. The cortico-basal ganglia-thalamo-cortical loop. Brain Res Brain Res Rev. 1995;20:91-127.

17. Misic B, Betzel RF, de Reus MA, van den Heuvel MP, Berman MG, Mclntosh AR et al. Network-level structure-function relationships in human neocortex. Cereb Cortex. 2016;26:3285-96.

18. Fox MD, Raichle ME. Spontaneous fluctuations in brain activity observed with functional magnetic resonance imaging. Nat Rev Neurosci. 2007;8:700-11

19. Allen EA, Erhardt EB, Damaraju E, Gruner W, Segall JM, Silva RF, et al. A baseline for the multivariate comparison of resting-state networks. Front Syst Neurosci. 2011;5:2.

20. Yeo BT, Krienen FM, Sepulcre J, Sabuncu MR, Lashkari D, Hollinshead M, et al. The organization of the human cerebral cortex estimated by intrinsic functional connectivity. J Neurophysiol. 2011;106:1125-65.

21. Smith SM, Fox PT, Miller KL, Glahn DC, Fox PM, Mackay CE, et al. Correspondence of the brain's functional architecture during activation and rest. Proc Natl Acad Sci USA. 2009;106:13040-5.

22. Chan MY, Alhazmi FH, Park DC, Savalia NK, Wig GS. Resting-state network topology differentiates task signals across the adult life span. J Neurosci. 2017;37:2734-45

23. Toulmin H, Beckmann CF, O'Muircheartaigh J, Ball G, Nongena P, Makropoulos A, et al. Specialization and integration of functional thalamocortical connectivity in the human infant. Proc Natl Acad Sci USA. 2015;112:6485-90.

24. Tu PC, Hsieh JC, Li CT, Bai YM, Su TP. Cortico-striatal disconnection within the cingulo-opercular network in schizophrenia revealed by intrinsic functional connectivity analysis: a resting fMRI study. Neuroimage. 2012;59:238-47.

25. Zhang D, Snyder AZ, Fox MD, Sansbury MW, Shimony JS, Raichle ME. Intrinsic functional relations between human cerebral cortex and thalamus. J Neurophysiol. 2008;100:1740-8.

26. Choi EY, Yeo BT, Buckner RL. The organization of the human striatum estimated by intrinsic functional connectivity. J Neurophysiol. 2012;108:2242-63.

27. Di Martino A, Scheres A, Margulies DS, Kelly AM, Uddin LQ, Shehzad Z, et al. Functional connectivity of human striatum: a resting state FMRI study. Cereb Cortex. 2008;18:2735-47.

28. Manoliu A, Riedl V, Doll A, Bauml JG, Muhlau M, Schwerthoffer D, et al. Insular dysfunction reflects altered between-network connectivity and severity of negative symptoms in schizophrenia during psychotic remission. Front Hum Neurosci. 2013;7:216

29. Baker JT, Holmes AJ, Masters GA, Yeo BT, Krienen F, Buckner RL, et al. Disruption of cortical association networks in schizophrenia and psychotic bipolar disorder. JAMA Psychiatry. 2014;71:109-18.

30. Crockett DJ. A comparison of empirically derived groups of aphasic patients on the Neurosensory Center Comprehensive Examination for Aphasia. J Clin Psychol. 1977;33:194-8.

31. Fett AKJ, Viechtbauer W, Dominguez MD, Penn DL, van Os J, Krabbendam L. The relationship between neurocognition and social cognition with functional outcomes in schizophrenia: a meta-analysis. Neurosci Biobehav R. 2011;35:573-88.

32. Kay SR, Fiszbein A, Opler LA. The positive and negative syndrome scale (PANSS) for schizophrenia. Schizophr Bull. 1987;13:261-76.

33. Beckmann CF, Smith SM. Probabilistic independent component analysis for functional magnetic resonance imaging. IEEE Trans Med Imaging. 2004;23:137-52.

34. Power JD, Mitra A, Laumann TO, Snyder AZ, Schlaggar BL, Petersen SE. Methods to detect, characterize, and remove motion artifact in resting state fMRI. Neuroimage. 2014;84:320-41.
35. Satterthwaite TD, Elliott MA, Gerraty RT, Ruparel K, Loughead J, Calkins ME, et al. An improved framework for confound regression and filtering for control of motion artifact in the preprocessing of resting-state functional connectivity data. Neuroimage. 2013;64:240-56.

36. Hutchison JL, Hubbard NA, Brigante RM, Turner M, Sandoval TI, Hillis GA, et al. The efficiency of $\mathrm{fMRI}$ region of interest analysis methods for detecting group differences. J Neurosci Methods. 2014;226: 57-65.

37. O'Reilly JX, Beckmann CF, Tomassini V, Ramnani N, Johansen-Berg H. Distinct and overlapping functional zones in the cerebellum defined by resting state functional connectivity. Cereb Cortex. 2010;20:953-65.

38. Jenkinson M, Bannister P, Brady M, Smith S. Improved optimization for the robust and accurate linear registration and motion correction of brain images. Neuroimage. 2002;17:825-41.

39. Dosenbach NU, Visscher KM, Palmer ED, Miezin FM, Wenger KK, Kang HC, et al. A core system for the implementation of task sets. Neuron. 2006;50:799-812.

40. Ham T, Leff A, de Boissezon X, Joffe A, Sharp DJ. Cognitive control and the salience network: an investigation of error processing and effective connectivity. J Neurosci. 2013;33:7091-8.

41. Menon V, Uddin LQ. Saliency, switching, attention and control: a network model of insula function. Brain Struct Funct. 2010;214:655-67.

42. Seeley WW, Menon V, Schatzberg AF, Keller J, Glover GH, Kenna H, et al. Dissociable intrinsic connectivity networks for salience processing and executive control. J Neurosci. 2007;27:2349-56.

43. Costafreda SG, Fu CH, Picchioni M, Toulopoulou T, McDonald C, Kravariti E. et al. Pattern of neural responses to verbal fluency shows diagnostic specificity for schizophrenia and bipolar disorder. BMC Psychiatry. 2011;11:18

44. Fu CHY, Morgan K, Suckling J, Williams SCR, Andrew C, Vythelingum GN, et al. A functional magnetic resonance imaging study of overt letter verbal fluency using a clustered acquisition sequence: greater anterior cingulate activation with increased task demand. Neuroimage. 2002;17:871-9.

45. Wagner G, De la Cruz F, Schachtzabel C, Gullmar D, Schultz CC, Schlosser RG, et al. Structural and functional dysconnectivity of the fronto-thalamic system in schizophrenia: a DCM-DTI study. Cortex. 2015;66:35-45.

46. Giraldo-Chica M, Rogers BP, Damon SM, Landman BA, Woodward ND. Prefrontalthalamic anatomical connectivity and executive cognitive function in schizophrenia. Biol Psychiatry. 2018;83:509-17.

47. Cheng W, Palaniyappan L, Li M, Kendrick KM, Zhang J, Luo Q, et al. Voxel-based, brain-wide association study of aberrant functional connectivity in schizophrenia implicates thalamocortical circuitry. NPJ Schizophr. 2015;1:15016.

48. Hoffman RE, Fernandez T, Pittman B, Hampson M. Elevated functional connectivity along a corticostriatal loop and the mechanism of auditory/verbal hallucinations in patients with schizophrenia. Biol Psychiatry. 2011;69:407-14.

49. Fox MD, Raichle ME. Spontaneous fluctuations in brain activity observed with functional magnetic resonance imaging. Nat Rev Neurosci. 2007;8:700-11.

50. Power JD, Cohen AL, Nelson SM, Wig GS, Barnes KA, Church JA, et al. Functional network organization of the human brain. Neuron. 2011;72:665-78.

51. Parent A, Hazrati LN. Functional anatomy of the basal ganglia. I. The cortico-basal ganglia-thalamo-cortical loop. Brain Res Brain Res Rev. 1995;20:91-127.

52. Bolam JP, Hanley JJ, Booth PA, Bevan MD. Synaptic organisation of the basal ganglia. J Anat. 2000;196(Pt 4):527-42.

53. Kumar VJ, van Oort E, Scheffler K, Beckmann CF, Grodd W. Functional anatomy of the human thalamus at rest. Neuroimage. 2017;147:678-91.

54. Kempf L, Nicodemus KK, Kolachana B, Vakkalanka R, Verchinski BA, Egan MF, et al Functional polymorphisms in PRODH are associated with risk and protection for schizophrenia and fronto-striatal structure and function. PLoS Genet. 2008;4: e1000252.

55. Krystal JH, Anticevic A, Yang GJ, Dragoi G, Driesen NR, Wang XJ, et al. Impaired tuning of neural ensembles and the pathophysiology of schizophrenia: a translational and computational neuroscience perspective. Biol Psychiatry. 2017;81:874-85.

56. Lewis DA, Curley AA, Glausier JR, Volk DW. Cortical parvalbumin interneurons and cognitive dysfunction in schizophrenia. Trends Neurosci. 2012;35:57-67.

57. Enwright JF, Sanapala S, Foglio A, Berry R, Fish KN, Lewis DA. Reduced labeling of parvalbumin neurons and perineuronal nets in the dorsolateral prefrontal cortex of subjects with schizophrenia. Neuropsychopharmacology. 2016;41:2206-14.

58. Shepherd GM. Corticostriatal connectivity and its role in disease. Nat Rev Neurosci. 2013;14:278-91.

59. Yang GJ, Murray JD, Wang XJ, Glahn DC, Pearlson GD, Repovs G, et al. Functional hierarchy underlies preferential connectivity disturbances in schizophrenia. Proc Natl Acad Sci USA. 2016:113:E219-228.

60. Haber SN, Calzavara R. The cortico-basal ganglia integrative network: the role of the thalamus. Brain Res Bull. 2009;78:69-74.

61. Hughes SW, Lorincz ML, Parri HR, Crunelli V. Infraslow $(<0.1 \mathrm{~Hz})$ oscillations in thalamic relay nuclei basic mechanisms and significance to health and disease states. Prog Brain Res. 2011;193:145-62. 
62. Kita $T$, Kita $H$. The subthalamic nucleus is one of multiple innervation sites for long-range corticofugal axons: a single-axon tracing study in the rat. J Neurosci. 2012;32:5990-9.

63. Mitchell AS. The mediodorsal thalamus as a higher order thalamic relay nucleus important for learning and decision-making. Neurosci Biobehav Rev. 2015;54:76-88.

64. Sherman SM. Thalamus plays a central role in ongoing cortical functioning. Nat Neurosci. 2016;19:533-41.

65. Lewis DA, Cruz DA, Melchitzky DS, Pierri JN. Lamina-specific deficits in parvalbumin-immunoreactive varicosities in the prefrontal cortex of subjects with schizophrenia: evidence for fewer projections from the thalamus. Am J Psychiatry. 2001;158:1411-22.

66. Howes OD, Kambeitz J, Kim E, Stahl D, Slifstein M, Abi-Dargham A, et al. The nature of dopamine dysfunction in schizophrenia and what this means for treatment. Arch Gen Psychiatry. 2012;69:776-86.

67. Cole DM, Oei NY, Soeter RP, Both S, van Gerven JM, Rombouts SA, et al. Dopamine-dependent architecture of cortico-subcortical network connectivity. Cereb Cortex. 2013;23:1509-16.
68. Sarpal DK, Robinson DG, Lencz T, Argyelan M, Ikuta T, Karlsgodt K, et al. Antipsychotic treatment and functional connectivity of the striatum in first-episode schizophrenia. JAMA Psychiatry. 2015;72:5-13.

69. Wang YC, Tang WJ, Fan XD, Zhang JY, Geng DY, Jiang KD, et al. Resting-state functional connectivity changes within the default mode network and the salience network after antipsychotic treatment in early-phase schizophrenia. Neuropsych Dis Treat. 2017;13:397-406.

70. Fox MD, Snyder AZ, Vincent JL, Corbetta M, Van Essen DC, Raichle ME. The human brain is intrinsically organized into dynamic, anticorrelated functional networks. Proc Natl Acad Sci U S A. 2005;102:9673-8.

71. Shehzad Z, Kelly AM, Reiss PT, Gee DG, Gotimer K, Uddin LQ, et al. The resting brain: unconstrained yet reliable. Cereb Cortex. 2009;19:2209-29.

72. Van Dijk KR, Sabuncu MR, Buckner RL. The influence of head motion on intrinsic functional connectivity MRI. Neuroimage. 2012;59:431-8.

73. Birn RM, Molloy EK, Patriat R, Parker T, Meier TB, Kirk GR, et al. The effect of scan length on the reliability of resting-state $\mathrm{fMRI}$ connectivity estimates. Neuroimage. 2013;83:550-8. 